\title{
BMJ Open Discordant Clostridioides difficile diagnostic assay and treatment practice: a cross-sectional study in a tertiary care hospital, Geneva, Switzerland
}

\author{
Lauriane Lenggenhager (D) ,, Marie-Céline Zanella (D) , ${ }^{1,3}$ Antoine Poncet (D) ,4 \\ Laurent Kaiser (D) ,1,2,5 Jacques Schrenzel (iD) 1,2,3
}

To cite: Lenggenhager $L$, Zanella M-C, Poncet A, et al. Discordant Clostridioides difficile diagnostic assay and treatment practice: a cross-sectional study in a tertiary care hospital, Geneva, Switzerland. BMJ Open 2020;10:e036342. doi:10.1136/ bmjopen-2019-036342

- Prepublication history for this paper is available online. To view these files, please visit the journal online (http://dx.doi org/10.1136/bmjopen-2019036342).

$\mathrm{LL}$ and M-CZ contributed equally.

Received 10 January 2020

Revised 20 April 2020

Accepted 10 July 2020

Check for updates

(c) Author(s) (or their employer(s)) 2020. Re-use permitted under CC BY-NC. No commercial re-use. See rights and permissions. Published by BMJ.

For numbered affiliations see end of article.

Correspondence to Dr Marie-Céline Zanella; marie-celine.zanella@hcuge.ch

\section{ABSTRACT}

Objectives To determine the proportion of patients who received a treatment for Clostridioides difficile infection (CDI) among those presenting a discordant $C$. difficile diagnostic assay and to identify patient characteristics associated with the decision to treat CDI.

Design Cross-sectional study.

Setting Monocentric study in a tertiary care hospital, Geneva, Switzerland.

Participants Among 4562 adult patients tested for C. difficile between March 2017 and March 2019, 208 patients with discordant tests' results (positive nucleic acid amplification test (NAAT+)/negative enzyme immunoassay (EIA-)) were included.

Main outcome measures Treatment for CDI.

Results CDI treatment was administered in 147 (71\%) cases. In multivariate analysis, an abdominal CT scan with signs of colitis (OR $14.7 ; 95 \% \mathrm{Cl} 1.96$ to 110.8 ) was the only factor associated with CDI treatment.

Conclusions The proportion of NAAT+/EIA- patients who received treatment questions the contribution of the EIA for the detection of toxin A/B after NAAT to limit overtreatment. Additional studies are needed to investigate if other factors are associated with the decision to treat.

\section{INTRODUCTION}

Clostridioides difficile (formerly Clostridium difficile) infection (CDI) is a toxin-mediated disease and the leading cause of healthcareassociated infection, as well as an increasing cause of community-associated diarrhoea. ${ }^{1-4}$ During the past decade, easy-to-perform and low-cost diagnostic tests have been developed, comprising nucleic acid amplification tests (NAATs) for the detection of toxin A/B genes and enzyme immunoassays (EIAs) for the detection of glutamate dehydrogenase $(\mathrm{GDH})$ and toxins $\mathrm{A} / \mathrm{B}$ in stool specimens. However, these tests are not recommended as stand-alone tests for CDI diagnosis due to their suboptimal sensitivity and specificity. ${ }^{56}$ European and US guidelines recommend a two-stage or three-stage diagnostic approach. ${ }^{5-9}$ This includes the use of a
Strengths and limitations of this study

- Patients were considered as treated for Clostridioides difficile infection (CDI) according to predefined criteria, including the appropriateness of the antibiotic treatment for CDI, timing of its introduction and duration, and the absence of any alternative justification for its prescription.

- Parameters investigated in multivariate analysis were limited to a selection of risk factors and clinical characteristics known to be associated with CDI.

- Patients without an indication for $C$. difficile testing were excluded from the study.

- Given the monocentric design of the study, our results may reflect local practice only in terms of the diagnostic algorithm and decision to treat.

- Given the observational design of the study and the routinely-collected origin of the data, some covariates may be missing in the model, thus leading to a risk for a phenomenon of confusion.

highly sensitive assay with a high negative predictive value (NPV), either NAAT or EIA for GDH (NPV of $99 \%-100 \%$ in a typical endemic situation with a prevalence of $5 \%$ ) and, if positive, a reflex test using a highly specific confirmatory assay with a high positive predictive value (PPV), typically a toxin A/B EIA (PPV of $98.5 \%) .^{5}$

CDI diagnosis relies on the association of clinical manifestations and microbiological tests documenting the presence of a toxigenic C. difficile strain and toxin/s in stools. ${ }^{10}$ Symptomatic patients with both tests positive (NAAT+ or GDH+/EIA+) are likely to suffer from CDI. In the presence of discordant results (NAAT+ or GDH+/EIA-), the EIA negative result may be interpreted either as a false-negative or a toxin level below threshold in the case of a patient effectively presenting with CDI or as a true negative in the case of $C$. difficile toxigenic strain carriage. A third-stage test, either NAAT, toxigenic culture or GDH, 
if not yet performed, can be performed to exclude a false-positive NAAT/GDH,${ }^{511}$ but will not distinguish CDI from toxigenic strain carriage. Therefore, this distinction in patients with discordant results relies on clinical evaluation, but current guidelines do not clearly state which factors should be taken into account. ${ }^{58}$

CDI overdiagnosis and subsequent overtreatment are major concerns regarding the emergence of resistance, particularly vancomycin-resistant Enterococcus spp. ${ }^{12}$ Although multiple-step algorithms have been recently implemented with the aim to avoid CDI overdiagnosis and subsequent overtreatment, the actual proportion of NAAT+/EIA- patients who receive a treatment for CDI remains poorly described, as well as the factors influencing the treatment decision. ${ }^{13}$

In this study, we aimed to identify the proportion of patients that receive a treatment for CDI among those with $C$. difficile discordant tests' results (NAAT+/EIA-) and patient characteristics associated with the decision to treat.

\section{METHODS}

\section{Study design, setting and population}

We conducted a cross-sectional study at Geneva University Hospitals, a 2000-bed Swiss tertiary care centre. Clinical and biological data (results of NAAT/EIA assays performed on stool samples) were collected from electronic medical records (EMR) and the hospital bacteriology laboratory, respectively. Inclusion criteria were all adult patients ( $\geq 18$ years) hospitalised or not, with $C$. difficile toxin assays performed on stool samples between 1 March 2017 and 1 March 2019 that yielded discordant results (NAAT+/EIA-). Exclusion criteria were asymptomatic patients (without diarrhoea, ileus or toxic megacolon), paediatric patients, patients with a treatment against $C$. difficile introduced $\geq 48$ hours before the results of tests, or without clinical data available in EMR form. In patients presenting several tests with discordant results over the study period, only the first test was considered for analysis.

\section{Outcomes and definitions}

The primary objective was to determine the proportion of adult patients with a first discordant test result who received a treatment for CDI and to identify patient characteristics and risk factors for CDI (if any) associated with CDI treatment. ${ }^{5}$

Patients were considered as treated for CDI if they fulfilled all of the following criteria: (1) an appropriate antibiotic treatment administered for CDI according to published guidelines: ${ }^{5814}$ (2) treatment introduced less than 48 hours before the results of tests; (3) treatment duration of $\geq 10$ days or still under treatment at the time of death and (4) treatment prescribed with a written decision in the EMR for CDI treatment, or without an alternative indication for its prescription. Of note, as faecal microbiota transplantation is not performed at our centre, it was not retained in the outcome definition.

In patients with a previous positive test (NAAT + or EIA+ orboth), only those who had received a treatment for CDI were considered as having a history of CDI. Abdominal CT scans were considered if they were performed less than 48 hours before and less than 10 days after the test result. Definitions of other characteristics and risks factors are described in the web-only online supplementary table S1.

\section{Laboratory methods}

Since 16 January 2017, the hospital bacteriology laboratory has implemented a two-step diagnostic algorithm comprising the use of a NAAT for $C$. difficile toxin B ( $T c d B$; BD MAX, Becton-Dickinson, Sparks, Maryland, USA), followed by an EIA for both toxins (A/B; XPect C. difficile Toxin A/B EIA, Remel, San Diego, California, USA) as a reflex confirmatory test if the NAAT is positive. Fresh stool samples collected in Cary-Blair tubes are delivered to the laboratory and processed immediately without restrictions related to stool consistency. Samples drawn at night or during the weekend are stored at $4^{\circ} \mathrm{C}$ in the laboratory before analysis. NAAT and EIA assays are performed daily from Monday to Saturday inclusive.

\section{Statistical analysis}

The decision was made to include all eligible patients, and no formal sample size calculation was performed. Instead, we restricted the number of investigated parameters before any confirmatory analysis. Based on the ' 10 events per variable' rule of thumb, we limited the number of parameters investigated to eight factors selected among known risk factors and clinical characteristics compatible with CDI. Patient characteristics and CDI risk factors were described overall and by treatment for CDI and reported as frequencies and percentages. A multivariate logistic regression model using a backward stepwise method was performed to determine which parameters were independently associated with CDI treatment. At each step, starting from all eight parameters, the variable with the highest $p$ value on the likelihood ratio test was removed from the model until all remaining factors were statistically significantly associated with CDI treatment at a twosided level of $5 \%$. Sensitivity analyses were performed to assess the robustness of the results when deceased patients were (a) excluded from the analysis and (b) considered as not treated. Missing data were systematically removed from analyses. Statistical significance was assessed at a two-sided 0.05 level for all analyses. All statistical analyses were performed using Stata software, V. 15 (StataCorp, College Station, Texas, USA).

\section{Patient and public involvement}

No patients were involved in the design, or conduct, or reporting, or dissemination of our research. The dissemination of the results to the included patients will not be performed. 
Tests for Clostridioides difficile performed on stool samples from 1 March 2017 to 1 March 2019

$\mathrm{N}=4562$ patients with 6931 tests

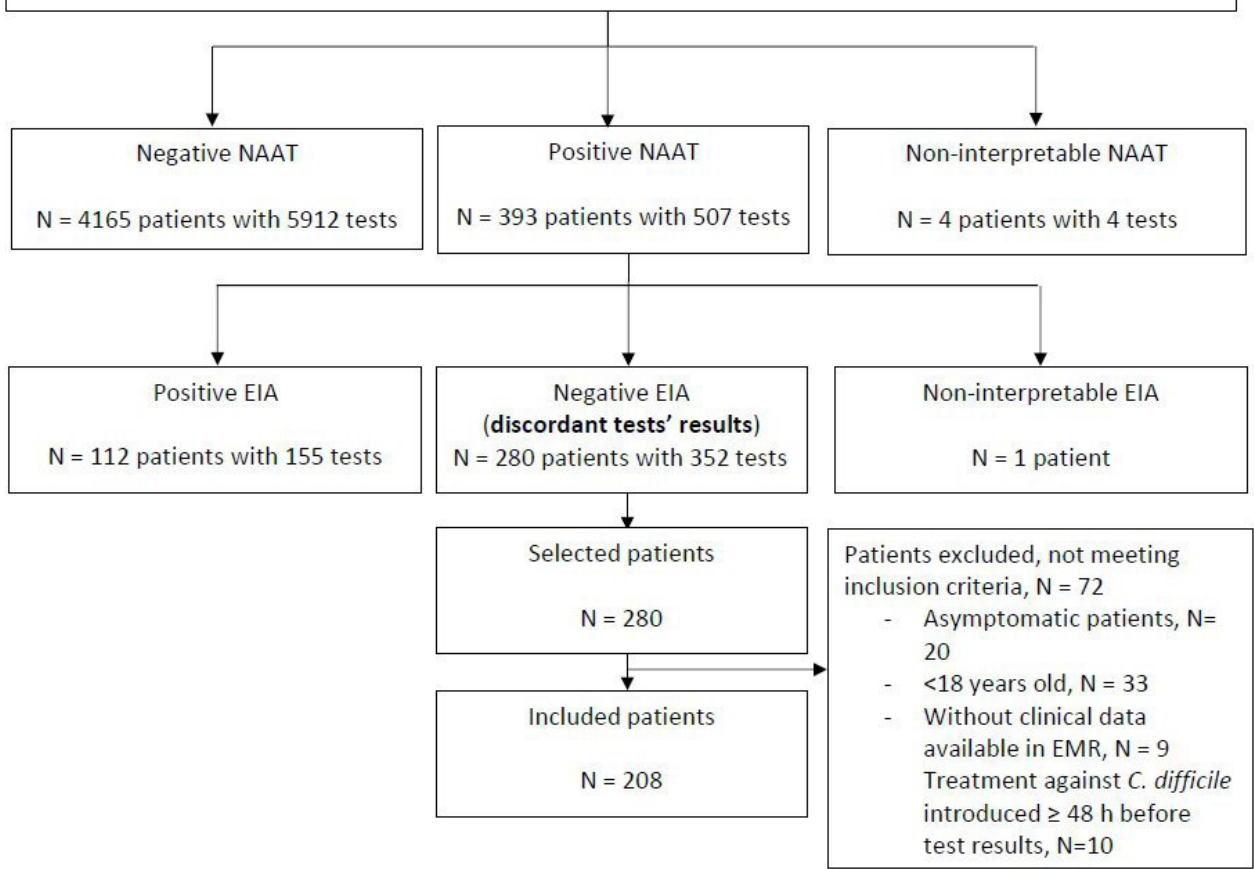

Figure 1 Flowchart of patient selection. EIA, enzyme immunoassay for toxin A/B; EMR, electronic medical records; NAAT, nucleic acid amplification test for toxin B.

\section{RESULTS}

\section{Patient characteristics}

During the study period, 4562 patients had at least one stool sample tested for C. difficile (corresponding to 6931 tests). A total of $393(8.6 \%)$ patients (corresponding to 507 tests) had NAAT+ samples; 280/393 (71.3\%; corresponding to 352 tests) had an EIA- for toxin A/B testing (NAAT+/EIA-). Two hundred and eighty $(6.1 \%)$ patients had $352(5.1 \%)$ discordant test results (figure 1). Among these, $72(25.7 \%)$ were excluded $(<18$ years $(n=33)$; asymptomatic patients $(\mathrm{n}=20)$; without available clinical data in the EMR, apart from demographics $(n=9]$ and with treatment against $C$. difficile introduced 48 hours or more before the results of tests $(n=10))$. We hereby analysed the first NAAT+/EIA- stool sample of the 208 patients included in the study (figure 1). Baseline patient characteristics are described in table 1 . Since the EIA confirmatory test is a reflex test after a NAAT + , the results of the two tests were available simultaneously in the patient's EMR. Median delay from prescription to results validation was 1 day (IQR $0-1$ ).

Among the 208 patients included, none presented with ileus or toxic megacolon, while an alternative diagnosis was reported in the EMR for six patients. One of five patients who underwent recto-sigmoidoscopy had typical endoscopic lesions and was treated. Fifty-nine patients (28\%) had an abdominal CT scan and 49 received a treatment for CDI (table 1). A CT scan was performed before the tests' results in 15/59 (25\%) patients and after results in 44 patients. The most frequent indications for the CT scan were: investigation for an abdominal infection (40\%); signs of colitis $(32 \%)$ and urological disease $(12 \%)$. Among patients with signs of colitis, a CT scan was performed to investigate CDI in $16(53 \%)$ patients.

\section{Treatment, treatment type and duration}

Overall, 147 patients $(71 \%)$ were treated for CDI. Treatment consisted of oral metronidazole for 132 patients $(90 \%)$ and oral vancomycin for 15 patients (10\%) (table 2). Treatment was initiated at the time of test results in 133 patients $(90 \%)$ and within the 48 hours preceding the results in the remaining 14 . Of the 145 treated patients with available data regarding severity criteria, 55 $(38 \%)$ presenting with severity criteria were treated for CDI (oral metronidazole $(n=46)$ and oral vancomycin $(\mathrm{n}=9))$. Among untreated patients $(\mathrm{n}=61), 46(75 \%)$ did not receive any CDI treatment and $15(25 \%)$ received a treatment for CDI during less than 10 days (median duration of treatment, 7 days; IQR, 4.5-8.5).

\section{Associated factors}

In univariate and multivariate analyses, abdominal CT scan with signs of colitis was the only associated factor with CDI treatment (OR 14.7; 95\% CI 1.96 to 110.8) (table 3).

\section{DISCUSSION}

In this study of patients who presented discordant test results (NAAT+/EIA-), $71 \%$ received a treatment for CDI, suggesting that most patients with discordant test 
Table 1 Baseline characteristics of included patients with NAAT+/EIA- $(n=208)$

\begin{tabular}{|c|c|c|c|c|}
\hline & All patients, no. (\%) & Treatment, no. (\%) & No treatment, no. (\%) & $P$ value \\
\hline & 208 & $147(71)$ & $61(29)$ & \\
\hline Age, mean (SD) & $66(19)$ & $67(19)$ & $64(20)$ & 0.309 \\
\hline Age $\geq 65$ years old ${ }^{\star}$ & $133(64)$ & $93(63)$ & $66(30)$ & 0.752 \\
\hline Gender, female n (\%) & $104(50)$ & $72(49)$ & $32(52)$ & 0.648 \\
\hline Hospitalisation*, n (\%) & $186(89)$ & $134(91)$ & $52(85)$ & 0.207 \\
\hline Internal medicine & $97(47)$ & $67(46)$ & $30(49)$ & \\
\hline Surgery & $39(19)$ & $25(17)$ & $14(23)$ & \\
\hline - Intensive care unit & $5(2)$ & $4(3)$ & $1(2)$ & \\
\hline Emergency & $17(8)$ & $15(10)$ & $2(3)$ & \\
\hline - Rehabilitation & $13(6)$ & $13(9)$ & 0 & \\
\hline - Oncology and haematology & $13(6)$ & $9(6)$ & $4(7)$ & \\
\hline - Gynaecology and obstetrics & $2(1)$ & $1(1)$ & $1(2)$ & \\
\hline \multicolumn{5}{|l|}{ Symptoms ${ }^{*}$} \\
\hline - Diarrhoea & 208 & $147(100)$ & $61(100)$ & \\
\hline \multicolumn{5}{|l|}{ - lleus } \\
\hline \multicolumn{5}{|l|}{ - Toxic megacolon } \\
\hline Presence of an alternative diagnosis in EMR & $6(3)$ & $1(1)$ & $5(8)$ & 0.009 \\
\hline Any severity criteriał & $72 / 205(35)$ & $55 / 145(38)$ & $17 / 60(28)$ & 0.19 \\
\hline Complicated*§ & 6/205 (3) & $5 / 145(3)$ & $1 / 60(2)$ & 0.673 \\
\hline - Sepsis & $4(2)$ & $4(3)$ & 0 & \\
\hline - Hypotension & $1(0.5)$ & $1(1)$ & 0 & \\
\hline Septic shock & $1(0.5)$ & 0 & $1(2)$ & \\
\hline Body mass index $\geq 30^{*}$ & 29/200 (15) & $21 / 142(15)$ & $8 / 58(14)$ & 0.856 \\
\hline Creatinine clearance $£ 60 \mathrm{~mL} / \mathrm{min}^{*}$ & $74 / 205(36)$ & $54 / 146(37)$ & 20/59 (34) & 0.677 \\
\hline Immunosuppressionף & $44(21)$ & $31(21)$ & $13(21)$ & 0.971 \\
\hline Abdominal imaging (CT) & $59(28)$ & $49(33)$ & $10(16)$ & 0.014 \\
\hline - Radiologic signs of colitis & $30(14)$ & $29(20)$ & $1(2)$ & 0.001 \\
\hline Ongoing PPI treatment ${ }^{\star}$ & $119 / 207(57)$ & $84 / 146(58)$ & $35(57)$ & 0.983 \\
\hline History of hospitalisation ${ }^{\star \star \star}$ & $196(94)$ & $139(95)$ & $57(93)$ & 0.75 \\
\hline History of CDI*†† & $19(9)$ & $12(8)$ & $7(11)$ & 0.45 \\
\hline History of antibiotic treatment* $\ddagger \ddagger$ & $137(66)$ & $96(65)$ & $41(67)$ & 0.792 \\
\hline Infectious disease specialist advice§§, n (\%) & $64(31)$ & $43(29)$ & $21(34)$ & 0.462 \\
\hline
\end{tabular}

${ }^{*}$ At the time of testing.

$\dagger \geq 3$ unformed stools in 24 hours.

$\ddagger$ Łlood leucocytes $>15 \mathrm{~g} / \mathrm{L}$ or serum creatinine $>133 \mu \mathrm{mol} / \mathrm{L}$.

§lleus, toxic megacolon, septic shock or hypotension.

ף|lncluding chemotherapy $\leq 60$ days before test prescription; SOT; HSCT; steroid (minimum $20 \mathrm{mg} /$ day prednisone or equivalent during at least

4 weeks before test prescription).

${ }^{*}$ Any hospitalisation of $\geq 48$ hours in the last 12 weeks before test prescription.

††History of positive test results in EMR (NAAT+/EIA+ orEIA+ orTC+).

$\ddagger \ddagger A n y$ antibiotic treatment of $\geq 48$ hours in the last 4 weeks before test prescription.

$\S \S A n y$ recommendation about treatment.

CDI, Clostridioides difficile infection; EIA, enzyme immunoassay; EMR, electronic medical record; HSCT, haematopoietic stem cell transplant;

NAAT, nucleic acid amplification test; PPI, proton pump inhibitor; SOT, solid organ transplant; TC, toxigenic culture.

results were considered as having a $\mathrm{CDI}$ and treated as such. These findings raise the question of the added value of EIA for CDI diagnosis. According to institutional guidelines at the time of the study, oral metronidazole was the most frequently administered antibiotic for patients without any severity criteria. ${ }^{5}$ Notably, $84 \%$ of treated patients with severity criteria were treated as nonsevere CDI, and these results highlight issues in treatment 
Table 2 Treatment type and duration

\begin{tabular}{lll} 
& No. (\%) \\
\hline CDI treatment, $\mathrm{n}(\%)$ & 147 & $(70.7)$ \\
$\begin{array}{l}\text { Metronidazole (oral) } \\
\text { Vancomycin (oral) }\end{array}$ & 132 & $(89.8)$ \\
$\begin{array}{l}\text { Median duration of treatment, } \\
\text { days (IQR) }\end{array}$ & 15 & $(10.2)$ \\
$\begin{array}{l}\text { Timing of CDI treatment introduction } \\
\text { Treatment introduced } \leq 48 \text { hour }\end{array}$ & 14 \\
$\quad \begin{array}{l}\text { prior to test results } \\
\text { Treatment introduced at the } \\
\quad \text { time of test results }\end{array}$ & 133 \\
\hline
\end{tabular}

CDI, Clostridioides difficile infection.

decisions in patients with discordant results and severity criteria for CDI. Results revealed that an abdominal CT scan with signs of colitis was significantly associated with CDI treatment in NAAT+/EIA- patients. Indeed, radiological signs of colitis are known as a convincing clue for active disease. ${ }^{1516}$

We did not demonstrate any association between a history of CDI and a past hospitalisation with CDI treatment. The proportion of patients with a history of CDI was lower among treated patients, but this result was not significant. These findings were surprising considering the risk of CDI recurrence after a previous CDI, and the risk of CDI associated with a history of hospitalisation. ${ }^{17-19}$ Concerning the presence of any severity criteria, we did not demonstrate any significant association with the decision to treat, although recent data revealed that leukocytosis and acute renal failure at presentation were associated with poor outcomes in patients with discordant results. $^{13}$

Although a positive EIA for toxin A/B has been associated with a more severe outcome, ${ }^{20} 21$ data are conflicting regarding the outcomes of patients with NAAT+/EIAresults. $^{13} 21$ When considering the suboptimal sensitivity of the currently available EIA tests for toxin A/B, clinicians mostly seemed to base their decision to treat patients with discordant results only on a NAAT + in order to avoid severe outcomes.

\section{LIMITATIONS}

This study has several limitations. First, it was monocentric, possibly reflecting local practice only. Second, the sample size limited the number of variables to investigate, as well as the capacity of the study to detect associations between the investigated factors and the outcome. Despite the fact that some are well-known risk factors associated with CDI, few were associated with the decision to treat, which may be due to a lack of power. Third, given the observational design, some covariates may be missing in the model, thus leading to a substantial risk for a phenomenon of confusion. Missing data may have resulted in information bias. Nevertheless, all main clinical characteristics and known risk factors for CDI according to current knowledge, were selected for univariate and multivariate analyses. Finally, one of the most important factors in the decision to treat that could not be analysed in the present study is human

Table 3 Univariate and multivariate regression models for the association of patient characteristics with CDI treatment $(n=208)$

\begin{tabular}{|c|c|c|c|c|c|c|}
\hline & \multicolumn{6}{|c|}{ Likelihood of receiving treatment for CDI } \\
\hline & \multicolumn{6}{|l|}{ OR (95\% Cl) } \\
\hline & $\begin{array}{l}\text { Treatment } n=147 \\
(70.7 \%)\end{array}$ & $\begin{array}{l}\text { No treatment } \\
n=61(29.3 \%)\end{array}$ & Unadjusted & $P$ value & Adjusted & $P$ value \\
\hline \multicolumn{7}{|l|}{ Characteristics } \\
\hline Age $\geq 65$ years & $93(63.3)$ & $40(65.6)$ & $0.9(0.48-1.69)$ & 0.752 & & \\
\hline Any severity criteria* & $55 / 145(37.9)$ & $17 / 60(28.3)$ & $1.54(0.8-2.97)$ & 0.192 & & \\
\hline Radiologic signs of colitis & $29(19.7)$ & $1(1.6)$ & $14.7(1.96-110.8)$ & 0.009 & $14.7(1.96-110.8)$ & 0.009 \\
\hline Ongoing PPI treatment & $84 / 146(57.5)$ & $35(57.4)$ & $1(0.54-1.84)$ & 0.983 & & \\
\hline History of hospitalisation $\ddagger$ & $139(94.6)$ & $57(93.4)$ & $1.21(0.35-4.2)$ & 0.754 & & \\
\hline History of CDIף & $12(8.2)$ & $7(11.5)$ & $0.68(0.25-1.83)$ & 0.452 & & \\
\hline
\end{tabular}

*Blood leucocytes count $>15 \mathrm{~g} / \mathrm{L}$ or serum creatinine $>133 \mu \mathrm{mol} / \mathrm{L}$.

†Including chemotherapy $\leq 60$ days before test prescription; SOT; HSCT; steroid (minimum $20 \mathrm{mg} /$ day prednisone or equivalent during at least 4 weeks before test prescription).

$\ddagger$ Any hospitalisation of $\geq 48$ hours in the last 12 weeks before test prescription.

§History of positive test results in EMR (NAAT+/EIA+ orEIA+ orTC+).

IAny antibiotic treatment of $\geq 48$ hour in the last 4 weeks before test prescription.

CDI, Clostridioides difficile infection ; EIA, enzyme immunoassay; EMR, electronic medical record; HSCT, hematopoietic stem cell transplant; NAAT, nucleic acid amplification test; PPI, proton pump inhibitor; SOT, solid organ transplant; TC, toxigenic culture. 
behaviour, which depends on the clinician's experience and each individual clinical situation.

Recent studies have questioned current algorithms for CDI diagnosis. Pollock et al revealed that the concentration of toxins A, B and A/B tested by a single molecule array were not significantly different in symptomatic (CDI) and asymptomatic (carriage) individuals selected on the basis of a positive NAAT for toxin gene, thus questioning the use of an EIA for toxin A/B after NAAT. ${ }^{22}$ By contrast, in patients selected on the basis of a positive toxin test, the concentrations were significantly higher in symptomatic patients, highlighting the possibility to prioritise toxin detection over toxin gene. ${ }^{22}$ C. difficile toxin gene realtime PCR cycle threshold values have been associated in some studies with toxin-EIA positive results and adverse outcomes. However, data are conflicting, and the accuracy of cycle threshold values for toxin-positive prediction remains low with currently available EIA assays. ${ }^{23}$ The use of a single ultrasensitive assay has been shown to be more sensitive and specific compared with a multistep algorithm using NAAT and EIA for toxin A/B. ${ }^{24}$

Regarding the missed opportunity of EIA to avoid overdiagnosis and CDI treatment as revealed by the proportion of treated patients with a negative EIA in our study, similar to Origuen et al, ${ }^{13}$ further investigations should be performed to assess the use of ultrasensitive and quantitative immunoassays for toxin $\mathrm{A} / \mathrm{B}$ detection as stand-alone tests for CDI diagnosis as evoked by recent studies described above.

\section{CONCLUSIONS}

In conclusion, $5.2 \%$ of patients tested for C. difficile harboured discordant C. difficile test results (NAAT+/ EIA-), with $71 \%$ receiving a treatment for CDI. An abdominal CT scan with signs of colitis was the only factor associated with the decision to treat. Nevertheless, additional studies are needed to assess whether other factors are associated with the decision to treat these patients. The proportion of NAAT+/EIA- patients that did not receive any treatment for CDI (29\%) questions the contribution of the EIA for the detection toxin A/B after NAAT to limit CDI overdiagnosis and overtreatment.

\section{Author affiliations \\ ${ }^{1}$ Division of Infectious Diseases, Geneva University Hospitals, Geneve, Switzerland ${ }^{2}$ University of Geneva Medical School, Geneve, Switzerland \\ ${ }^{3}$ Laboratory of Bacteriology, Division of Laboratory Medicine, Geneva University Hospitals, Geneva, Switzerland \\ ${ }^{4}$ Division of Clinical Epidemiology, Geneva University Hospitals, Geneve, Switzerland ${ }^{5}$ Laboratory of Virology, Division of Laboratory Medicine and Division of Infectious Diseases, Geneva University Hospitals, Geneva, Switzerland}

Acknowledgements The authors thank Rosemary Sudan for editorial assistance.

Contributors LL, M-CZ, AP and JS contributed to the conception and design of the study, advised on all statistical aspects and interpreted the data. LL and M-CZ performed the statistical analysis, assisted by AP. LL, M-CZ, AP, LK and JS drafted and reviewed the manuscript and approved the final version to be published. LL, $\mathrm{M}-\mathrm{CZ}$ and $\mathrm{JS}$ had full access to all of the data in the study and take responsibility for the integrity of the data and the accuracy of the data analysis. LL and M-CZ contributed equally to this work and are joint first authors. LL, M-CZ and JS are the guarantors. The corresponding author attests that all listed authors meet authorship criteria and that no others meeting the criteria have been omitted. The corresponding author has the right to grant on behalf of all authors and does grant on behalf of all authors, a worldwide licence to the publishers and its licensees in perpetuity, in all forms, formats and media (whether known now or created in the future), to (1) publish, reproduce, distribute, display and store the contribution, (2) translate the contribution into other languages, create adaptations, reprints, include within collections and create summaries, extracts and/or, abstracts of the contribution, (3) create any other derivative work(s) based on the contribution, (4) to exploit all subsidiary rights in the contribution, (5) the inclusion of electronic links from the contribution to third party material where-ever it may be located and (6) licence any third party to do any or all of the above.

Funding The authors have not declared a specific grant for this research from any funding agency in the public, commercial or not-for-profit sectors.

Competing interests All authors have completed the ICMJE uniform disclosure form at www.icmje.org/coi_disclosure.pdf and declare: no support from any organisation for the submitted work; no financial relationships with any organisations that might have an interest in the submitted work in the previous three years; no other relationships or activities that could appear to have influenced the submitted work.

Patient and public involvement Patients and/or the public were not involved in the design, or conduct, or reporting, or dissemination plans of this research.

Patient consent for publication Not required.

Ethics approval The study was approved by the Geneva ethics commission and a waiver of informed consent was granted due to its retrospective nature (study number 2018-02012).

Provenance and peer review Not commissioned; externally peer reviewed.

Data availability statement Data may be obtained from a third party and are not publicly available. Extra data can be accessed via the Dryad data repository at http://datadryad.org/ with the doi: 10.5061/dryad.jm63xsj7r.

Open access This is an open access article distributed in accordance with the Creative Commons Attribution Non Commercial (CC BY-NC 4.0) license, which permits others to distribute, remix, adapt, build upon this work non-commercially, and license their derivative works on different terms, provided the original work is properly cited, appropriate credit is given, any changes made indicated, and the use is non-commercial. See: http://creativecommons.org/licenses/by-nc/4.0/.

\section{ORCID iDs}

Lauriane Lenggenhager http://orcid.org/0000-0001-8669-643X

Marie-Céline Zanella http://orcid.org/0000-0001-9544-1295

Antoine Poncet http://orcid.org/0000-0003-0998-853X

Laurent Kaiser http://orcid.org/0000-0002-0857-2252

Jacques Schrenzel http://orcid.org/0000-0001-5464-7764

\section{REFERENCES}

1 Fawley WN, Davies KA, Morris T, et al. Enhanced surveillance of Clostridium difficile infection occurring outside hospital, England, 2011 to 2013. Euro Surveill 2016;2.

2 Freeman J, Bauer MP, Baines SD, et al. The changing epidemiology of Clostridium difficile infections. Clin Microbiol Rev 2010;23:529-49.

3 Smits WK, Lyras D, Lacy DB, et al. Clostridium difficile infection. Nat Rev Dis Primers 2016;2:16020.

4 Kuehne SA, Cartman ST, Heap JT, et al. The role of toxin A and toxin B in Clostridium difficile infection. Nature 2010;467:711-3.

5 Crobach MJT, Planche T, Eckert C, et al. European society of clinical microbiology and infectious diseases: update of the diagnostic guidance document for Clostridium difficile infection. Clin Microbiol Infect 2016;22:S63-81.

6 Burnham C-AD, Carroll KC. Diagnosis of Clostridium difficile infection: an ongoing conundrum for clinicians and for clinical laboratories. Clin Microbiol Rev 2013;26:604-30.

7 Gateau C, Couturier J, Coia J, et al. How to: diagnose infection caused by Clostridium difficile. Clin Microbiol Infect 2018;24:463-8.

8 McDonald LC, Gerding DN, Johnson S, et al. Clinical practice guidelines for Clostridium difficile infection in adults and children: 2017 update by the infectious diseases Society of America (IDSA) and Society for healthcare epidemiology of America (SheA). Clin Infect Dis 2018;66:e1-48. 
9 Guery B, Galperine T, Barbut F. Clostridioides difficile: diagnosis and treatments. BMJ 2019;366:14609.

10 Leffler DA, Lamont JT. Clostridium difficile infection. N Engl J Med 2015;372:1539-48.

11 Crobach MJT, Baktash A, Duszenko N, et al. Diagnostic guidance for C. difficile infections. Adv Exp Med Biol 2018;1050:27-44.

12 Stevens VW, Khader K, Echevarria K, et al. Use of oral vancomycin for Clostridioides difficile infection and the risk of vancomycinresistant enterococci. Clin Infect Dis 2020;71:645-51.

13 Origüen J, Corbella L, Orellana M Á, et al. Comparison of the clinical course of Clostridium difficile infection in glutamate dehydrogenasepositive toxin-negative patients diagnosed by PCR to those with a positive toxin test. Clin Microbiol Infect 2018;24:414-21.

14 Ooijevaar RE, van Beurden YH, Terveer EM, et al. Update of treatment algorithms for Clostridium difficile infection. Clin Microbiol Infect 2018;24:452-62.

15 Bartlett JG, Gerding DN. Clinical recognition and diagnosis of Clostridium difficile infection. Clin Infect Dis 2008;46:S12-18.

16 Kirkpatrick ID, Greenberg HM. Evaluating the CT diagnosis of Clostridium difficile colitis: should CT guide therapy? AJR Am J Roentgenol 2001;176:635-9.

17 Deshpande A, Pasupuleti V, Thota P, et al. Risk factors for recurrent Clostridium difficile infection: a systematic review and meta-analysis. Infect Control Hosp Epidemiol 2015;36:452-60.
18 Shivashankar R, Khanna S, Kammer PP, et al. Clinical predictors of recurrent Clostridium difficile infection in out-patients. Aliment Pharmacol Ther 2014;40:518-22.

19 Bignardi GE. Risk factors for Clostridium difficile infection. J Hosp Infect 1998;40:1-15.

20 Planche TD, Davies KA, Coen PG, et al. Differences in outcome according to Clostridium difficile testing method: a prospective multicentre diagnostic validation study of $\mathrm{C}$ difficile infection. Lancet Infect Dis 2013;13:936-45.

21 Polage CR, Gyorke CE, Kennedy MA, et al. Overdiagnosis of Clostridium difficile infection in the molecular test era. JAMA Intern Med 2015;175:1792-801.

22 Pollock NR, Banz A, Chen X, et al. Comparison of Clostridioides difficile stool toxin concentrations in adults with symptomatic infection and asymptomatic carriage using an ultrasensitive quantitative immunoassay. Clin Infect Dis 2019;68:78-86.

23 Sandlund J, Wilcox MH. Ultrasensitive detection of Clostridium difficile toxins reveals suboptimal accuracy of toxin gene cycle thresholds for toxin predictions. J Clin Microbiol 2019;57

24 Sandlund J, Bartolome A, Almazan A, et al. Ultrasensitive detection of Clostridioides difficile toxins $\mathrm{A}$ and $\mathrm{B}$ by use of automated single-molecule counting technology. J Clin Microbiol 2018;56:e00908-18. 